\title{
ARTIGO ORIGINAL Duração do sono em adolescentes de diferentes níveis socioeconômicos
}

\author{
Sleep duration in adolescents of different socioeconomic status
}

Maria Perpeto Socorro Leite Bernardo', Érico Felden Pereira², Fernando Mazzilli Louzada², Vânia D'Almeida'

\section{Palavras-chave}

Sono, privação do sono, adolescente, saúde escolar.

\section{Keywords}

Sleep, sleep deprivation, adolescent, school health.

\section{RESUMO}

Objetivo: Investigar a duração de sono na adolescência em diferentes níveis socioeconômicos. Método: Foram investigados 863 adolescentes de 10 a 19 anos em duas escolas de São Paulo, SP, Brasil. As coletas foram realizadas por meio de questionários para identificação de informações sobre os hábitos de sono e nível socioeconômico. Resultados: A duração média de sono nos dias da semana foi de 8,83(1,87) horas e a prevalência de adolescentes com duração de sono de oito ou menos horas diárias foi de 39,0\% nos dias com aula. Adolescentes da classe baixa apresentaram menor duração do sono $(p=0,043)$. Na análise ajustada, a idade, o nível socioeconômico e o hábito de tirar a sesta foram os principais fatores associados a poucas horas de sono. Os participantes de 18 a 19 anos apresentaram maior prevalência de poucas horas de sono em comparação aos de 10 a 11 anos ( $P R=4,78$; C195\%: 1,98-11,53), assim como os adolescentes da classe alta em comparação com a classe baixa ( $P R=1,48 ; C 195 \%: 1,20-1,83)$. Conclusão: Os resultados mostraram associações entre o nível socioeconômico e os hábitos de sono de adolescentes.

\begin{abstract}
Objective: To investigate the sleep duration in adolescents of different socioeconomic status. Method: We investigated 863 adolescents from 10 to 19 years in two schools in São Paulo, SP, Brazil. Sleep habits data and socioeconomic status were obtained by questionnaires. Results: Mean sleep duration on days of the week was 8.83(1.87) hours and the prevalence of adolescents with eight or fewer hours/day was 39.0\% on school days ( $p=0.043)$. On adjusted analysis, age, socioeconomic status and taking a nap habit were the main factors associated with few hours of sleep. Older students (aged 18 to 19 years) showed higher prevalence of few hours of sleep when compared to younger students (10 to 11 years) ( $R P=4.78$; IC95\%: 1.98-11.53), as well as for upper class adolescents when compared to those with lower socioeconomic status. Conclusion: Results showed the association between socioeconomic status and adolescents' sleep/wake habits.
\end{abstract}

1 Universidade Federal de São Paulo (Unifesp).

2Universidade Federal do Paraná (UFPR).

Recebido em

2/10/2009

Aprovado em

10/12/2009

Endereço para correspondência: Érico Felden Pereira

Rua Otacílio Chaves, 253, Nossa Senhora do Perpétuo Socorro - 97045-360 - Santa Maria, RS

E-mail: ericofelden@gmail.com 


\section{INTRODUÇÃO}

As relações entre o contexto socioambiental com os diversos desfechos em saúde e o comportamento das variáveis biológicas é tema contemporâneo nas investigações em saúde coletiva. No Brasil, a discussão sobre as desigualdades em saúde vem ganhando destaque, principalmente, a partir de resultados de investigações populacionais que identificaram um aumento nas prevalências de doenças crônicodegenerativas, especialmente nas populações com baixo nível socioeconômico. A má alimentação, o baixo nível de atividade física e a dificuldade de acesso a serviços de saúde de qualidade, entre outros, são apontados como possíveis causas dessa associação ${ }^{1,2}$.

No contexto das desigualdades em saúde, os estudos sobre sono têm focado suas análises no comportamento de diferentes etnias, analisando, normalmente, a presença de dissonias ${ }^{3}$. Os principais estudos, neste contexto, foram realizados com crianças e adolescentes norte-americanos com diferentes origens étnicas, normalmente, comparando origens africanas e europeias e têm verificado uma tendência de jovens com menor nível socioeconômico apresentarem pior qualidade de sono, maiores prevalências de insônia, menor duração do sono, maior frequência de despertares noturnos e apneia ${ }^{4-11}$.

A adolescência é uma fase de vida marcada por mudanças biopsicossociais importantes, inclusive em relação ao padrão do ciclo vigília-sono. Na puberdade, pode ser identificado um atraso de fase, caracterizado por horários tardios de dormir e acordar que, somados aos horários sociais e de início das aulas pela manhã, levam a uma importante diminuição das horas de sono ${ }^{12-14}$. Por conseguinte, uma duração inadequada de sono está associada a um aumento da sonolência diurna, dificuldades de aprendizagem, diminuição da qualidade de vida e maiores prevalências de doenças como a obesidade ${ }^{7,15,16}$

A literatura ${ }^{17-18}$ aponta que as atividades sociais e hábitos em geral têm apresentado um deslocamento para horários cada vez mais noturnos, enquanto as aulas têm começado cada vez mais cedo, levando a uma importante diminuição das horas de sono e um persistente débito de sono no decorrer da semana. Além da escassez de estudos que tratem de comportamentos do sono relacionados aos fatores socioeconômicos em crianças e adolescentes nos países em desenvolvimento, não foram encontradas análises da tendência de duração do sono no decorrer da adolescência nos diferentes níveis socioeconômicos. Dessa forma, objetivou-se neste estudo investigar o comportamento da duração do sono de adolescentes de 10 a 19 anos de diferentes níveis socioeconômicos.

\section{MÉTODOS}

O estudo foi conduzido entre agosto e novembro de 2005 com adolescentes residentes em São Paulo, SP, Brasil, e trata-se de uma pesquisa de cunho transversal. A amostra foi composta de 863 crianças e adolescentes de área urbana, estudantes de duas escolas, uma no centro da cidade e outra em região periférica. Foram incluídos no estudo estudantes do sexo masculino e feminino das quatro últimas séries do ensino fundamental e das duas primeiras séries do ensino médio (idades entre 10 e 19 anos). Um total de 463 estudantes da Escola A (EECMA) e 400 da Escola B (EEMLAMR) participaram do estudo. Foram incluídos na amostra os alunos com idades de 10 a 19 anos, que estavam presentes em sala de aula no momento das coletas, preencheram adequadamente os instrumentos de pesquisa, aceitaram e foram autorizados a participar da amostra.

\section{Procedimentos}

Este estudo foi aprovado pelo Comitê de Ética em Pesquisa da Universidade Federal de São Paulo (CEP \# 0857/04). Todos os estudantes das duas escolas foram convidados a participar das coletas de forma voluntária e com autorização dos responsáveis. Os participantes responderam a um questionário de hábitos de sono e um de avaliação socioeconômica. Os questionários foram aplicados dentro da sala de aula, depois de o consentimento ter sido obtido das autoridades escolares. Após uma breve explicação sobre o objetivo do estudo, os estudantes levaram entre 15 e 30 minutos para responder aos questionários.

\section{Avaliações}

Os comportamentos relacionados ao sono foram coletados por meio de um questionário ${ }^{19} \mathrm{com}$ questões sobre a existência de algum problema de saúde e a ocorrência de um possível distúrbio de sono. A duração do sono foi identificada considerando os horários de dormir e acordar durante os dias com e sem aula. O nível socioeconômico foi avaliado seguindo as recomendações propostas pela Associação Brasileira de Empresas de Pesquisa (ABEP) ${ }^{20}$, que considera a posse de bens móveis e o grau de escolaridade do chefe da família, classificando os adolescentes nas classes A1, A2, B1, B2, C, D e E. Para esse estudo, considerou-se classe alta (A1, $A 2$, B1 e B2), média (C) e baixa ( $D$ e E).

\section{Análise estatística}

As diferenças entre a duração do sono considerando idade, escolas, gênero e classes sociais foram analisadas por meio do teste Kruskal-Wallis, sendo complementado pelo teste de comparações múltiplas de Dunn, quando se observou diferença estatisticamente significante. Para a identificação de diferenças entre proporções nas variáveis categóricas, foi aplicado o teste Qui-Quadrado. Com o objetivo de analisar as variáveis associadas à duração de sono de oito ou menos horas, controlando possíveis fatores de confusão, foi utilizada a regressão de Poisson ${ }^{21}$ considerando como variáveis independentes: idade, sexo, escola, nível socioeconômico, turno 
escolar, trabalho e sesta. As variáveis selecionadas para o modelo ajustado foram aquelas que apresentaram $p<0,25$ no teste do Qui-Quadrado ${ }^{22}$. Adotou-se, em todas as análises estatísticas, um nível de probabilidade de significância de 5\%.

\section{RESULTADOS}

A duração média de sono nos dias com aula foi de $8,83(1,87)$ horas. A prevalência de participantes com duração de sono de oito ou menos horas foi de 39,0\% nos dias com aula e $23,2 \%$ nos dias sem aula (Tabela 1). Não foram observadas diferenças significativas na duração do sono entre os sexos nos dias com aula $(p=0,111)$ e sem aula $(p=0,551)$. Da mesma forma, não foram observadas diferenças na duração do sono entre as escolas A e B nos dias com aula $(p=0,175)$ e nos dias sem aula $(p=0,177)$

Tabela 1. Resultados descritivos das variáveis investigadas

\begin{tabular}{|c|c|}
\hline Idade, anos & $\begin{array}{l}\text { Frequência* } \\
14,47(2,10)\end{array}$ \\
\hline \multicolumn{2}{|l|}{ Faixas etárias (anos) } \\
\hline $10-11$ & 12,0 \\
\hline $12-13$ & 21,2 \\
\hline $14-15$ & 28,6 \\
\hline $16-17$ & 32,8 \\
\hline $18-19$ & 5,5 \\
\hline Masculino, \% & 47,4 \\
\hline Escola A (\%) & 53,5 \\
\hline Duração do sono nos dias com aula, horas & $8,82(1,87)$ \\
\hline Duração do sono nos dias sem aula, horas & $9,60(1,97)$ \\
\hline \multicolumn{2}{|l|}{ Duração do sono nos dias com aula, \% } \\
\hline$<6$ & 4,6 \\
\hline 6-8 & 34,4 \\
\hline$>8$ & 61,0 \\
\hline \multicolumn{2}{|l|}{ Duração do sono nos dias sem aula, \% } \\
\hline$<6$ & 3,5 \\
\hline 6-8 & 19,6 \\
\hline$>8$ & 76,9 \\
\hline \multicolumn{2}{|l|}{ Classe socioeconômica } \\
\hline Alta & 41,7 \\
\hline Média & 43,6 \\
\hline Baixa & 14,7 \\
\hline \multicolumn{2}{|l|}{ Turno escolar } \\
\hline Manhã & 18,9 \\
\hline Tarde & 50,6 \\
\hline Noite & 30,5 \\
\hline Trabalho, \% & 18,4 \\
\hline \multicolumn{2}{|l|}{ Sesta } \\
\hline Nunca & 42,0 \\
\hline Às vezes & 52,8 \\
\hline Sempre & 5,2 \\
\hline Horário médio de dormir com aula & $22,93(1,59)$ \\
\hline Horário médio de dormir sem aula & $24,03(2,30)$ \\
\hline Horário médio de acordar com aula & $7,81(1,85)$ \\
\hline Horário médio de acordar sem aula & $9,67(1,75)$ \\
\hline
\end{tabular}

* Os valores foram expressos em média (desvio-padrão) ou porcentagem.
As comparações entre as classes socioeconômicas mostraram diferenças significativas na duração do sono nos dias com aula ( $p=0,043)$, no horário de dormir nos dias sem aula $(p=0,033)$ e no horário de acordar nos dias com aula $(p=0,040)$ (Figuras 1, 2 e 3). As análises de comparações múltiplas mostraram diferenças na duração do sono entre as classes baixa e média e baixa e alta $(p<0,005)$ durante os dias com aula e a duração do sono apresentou tendência de diminuição com o aumento do nível socioeconômico (Figura 1). Os jovens da classe alta dormiam mais tarde em relação aos da classe baixa nos dias sem aula $(p<0,005)$, não sendo observadas diferenças considerando a classe média (Figura 2). As classes baixa e média apresentaram comportamentos diferenciados em relação ao horário de acordar nos dias com aula, sendo que os jovens de classe média acordam mais cedo $(p<0,005)$, conforme mostra a figura 3. Na comparação da duração do sono entre jovens

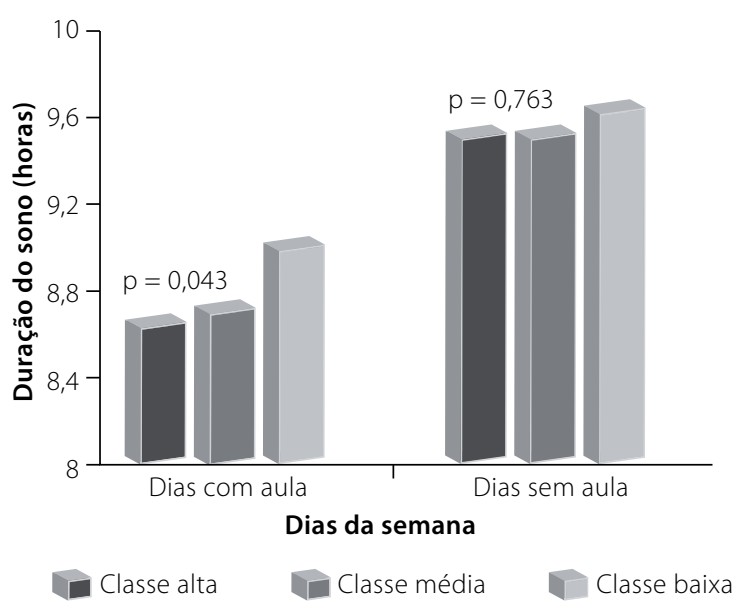

Figura 1. Duração do sono de acordo com o nível socioeconômico.

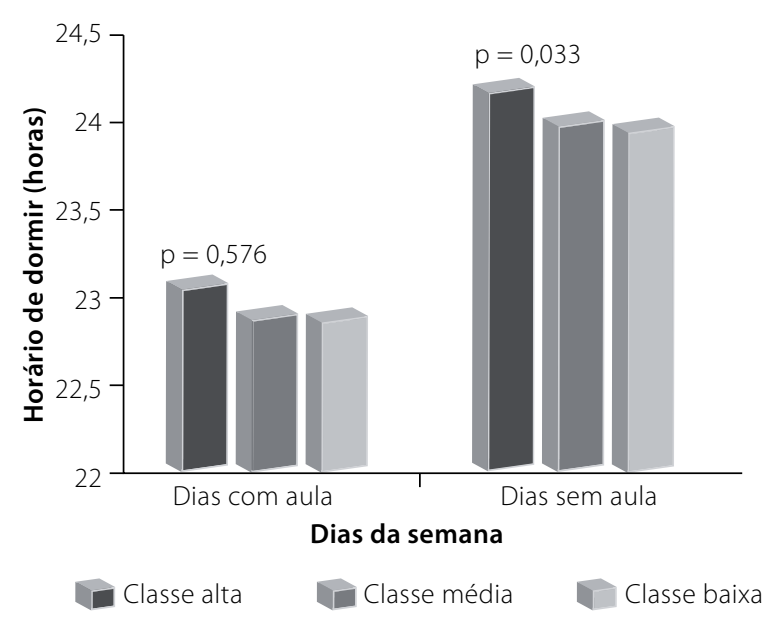

Figura 2. Horário de dormir de acordo com o nível socioeconômico. 
trabalhadores e não trabalhadores (Figura 4) foram observadas diferenças significativas considerando as classes baixa $(p<0,001)$ e média $(p=0,011)$, e tais diferenças não se mantiveram analisando-se a duração do sono nos fins de semana.

As análises de atraso no horário de dormir e da diminuição das horas de sono com o avanço da idade na adolescência, considerando o nível socioeconômico, foram apresentadas nas figuras 5 e 6 , que mostraram uma tendência de diminuição das horas de sono e um atraso no horário de dormir menos evidente, especialmente no final da puberda-

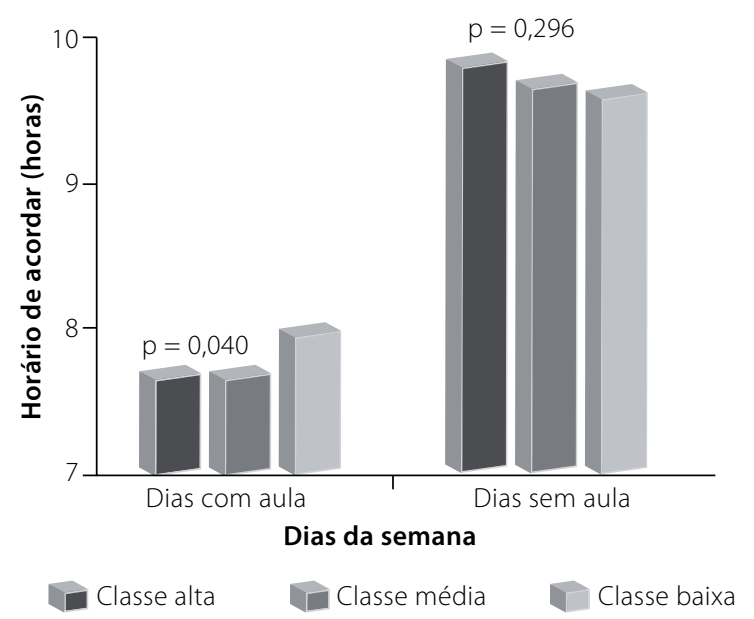

Figura 3. Horário de acordar de acordo com o nível socioeconômico.

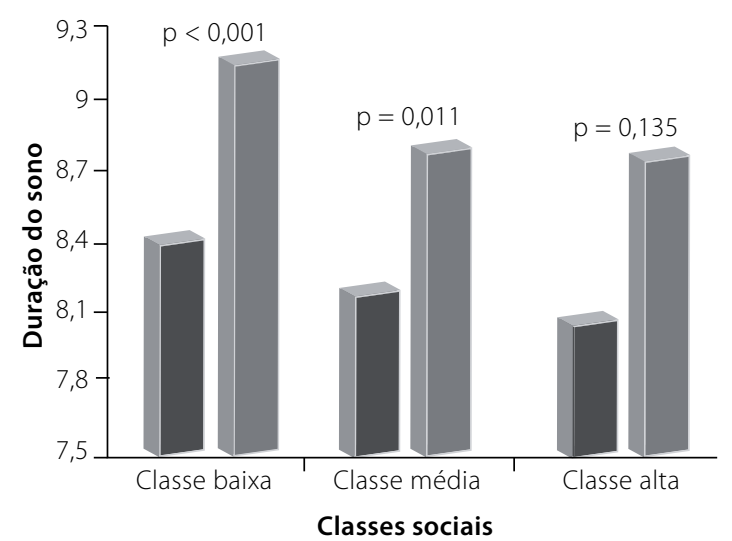

Trabalhadores

Não trabalhadores

Figura 4. Duração do sono nos dias com aula de adolescentes trabalhadores e não trabalhadores de acordo com o nível socioeconômico. de, na classe baixa. A análise de razões de prevalências (Tabela 2) mostrou que os jovens da classe alta apresentaram prevalências de oito ou menos horas de sono 1,48 vez maior que os da classe baixa. Na análise ajustada, a idade, o nível socioeconômico, o turno escolar e o hábito de tirar a sesta apresentaram-se como fatores associados a poucas horas de sono. Os adolescentes de 18 a 19 anos apresentaram maior prevalência de poucas horas de sono em comparação aos de 10 a 11 anos ( $P R=4,78$; C195\%: 1,98-11,53), assim como os adolescentes da classe alta em comparação com a classe baixa (PR =1,48; C195\%: 1,20-1,83).

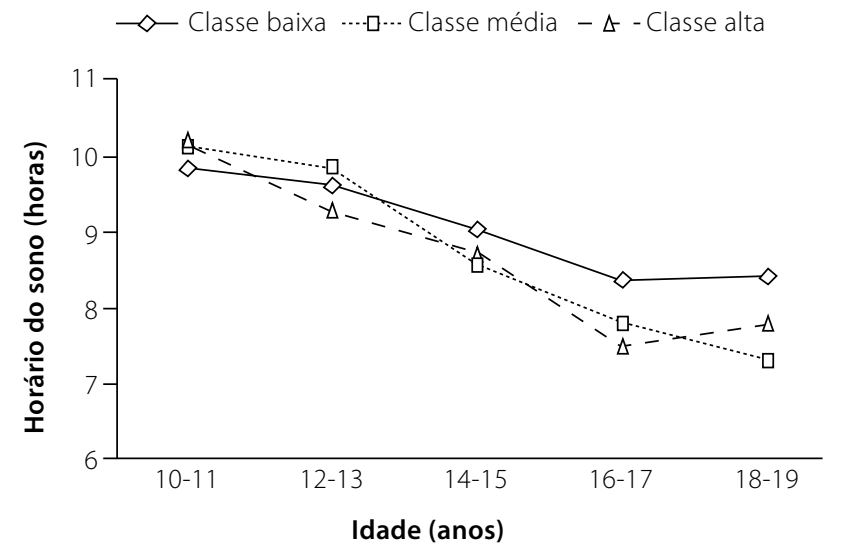

Figura 5. Tendência de diminuição das horas de sono de acordo com a idade.

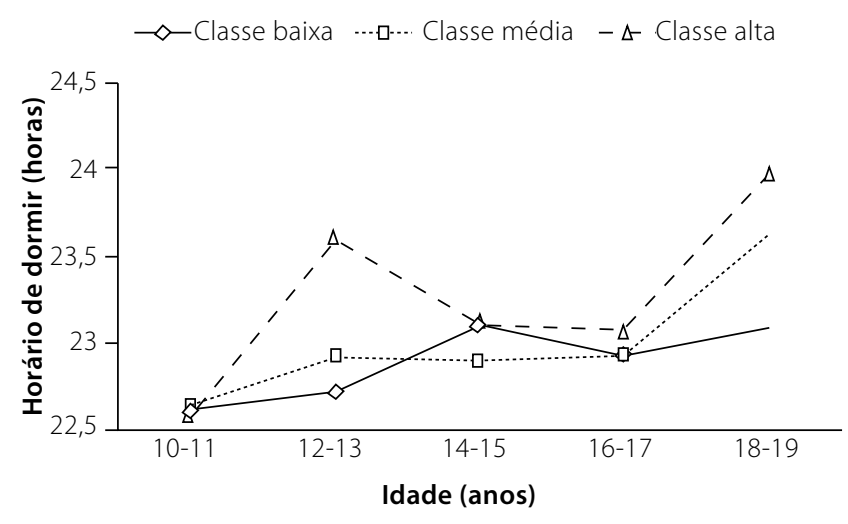

Figura 6. Tendência de atraso de fase de acordo com a idade. 
Tabela 2. Razões de prevalências (RP) utilizando-se como variável dependente 8 ou menos horas de sono nos dias com aula

\begin{tabular}{|c|c|c|c|c|c|}
\hline \multirow{2}{*}{ Variáveis } & \multirow{2}{*}{ Prevalência (\%) } & \multicolumn{2}{|c|}{ Análise não ajustada ${ }^{\mathrm{a}}$} & \multicolumn{2}{|c|}{ Análise ajustada ${ }^{b}$} \\
\hline & & $\operatorname{RP}(1 \mathrm{CO5} \%)$ & $p$ & $\operatorname{RP}(1 \mathrm{CO5} \%)$ & $\mathrm{p}$ \\
\hline \multicolumn{6}{|l|}{ Idade (anos) } \\
\hline $10-11$ & 5,82 & 1,00 & $<0,001$ & 1,00 & $<0,001$ \\
\hline $12-13$ & 14,83 & $2,55(1,08-5,94)$ & & $2,77(1,17-6,52)$ & \\
\hline $14-15$ & 42,86 & $7,36(3,29-16,46)$ & & $4,43(1,93-10,19)$ & \\
\hline $16-17$ & 57,65 & $9,90(4,62-22,15)$ & & $4,56(1,96-10,61)$ & \\
\hline $18-19$ & 59,57 & $10,23(4,50-22,80)$ & & $4,78(1,98-11,53)$ & \\
\hline \multicolumn{6}{|l|}{ Sexo } \\
\hline Masculino & 37,84 & 1,00 & 0,862 & Excluído & - \\
\hline Feminino & 38,58 & $1,02(0,86-1,20)$ & & & \\
\hline \multicolumn{6}{|l|}{ Escola } \\
\hline B & 33,33 & 1,00 & 0,008 & 1,00 & 0,143 \\
\hline A & 42,48 & $1,27(1,60-1,50)$ & & $1,07(0,91-1,26)$ & \\
\hline \multicolumn{6}{|c|}{ Nível socioeconômico } \\
\hline Classe baixa & 30,36 & 1,00 & $<0,001$ & 1,00 & 0,009 \\
\hline Classe média & 41,80 & $1,38(1,12-1,64)$ & & $1,22(1,03-1,46)$ & \\
\hline Classe alta & 48,76 & $1,61(1,22-1,96)$ & & $1,48(1,20-1,83)$ & \\
\hline \multicolumn{6}{|l|}{ Turno } \\
\hline Manhã & 63,36 & $1,10(0,93-1,28)$ & $<0,001$ & $1,02(0,85-1,22)$ & 0,039 \\
\hline Tarde & 15,90 & $0,28(0,21-0,35)$ & & $0,43(0,31-0,60)$ & \\
\hline Noite & 57,40 & 1,00 & & 1,00 & \\
\hline \multicolumn{6}{|l|}{ Trabalho } \\
\hline Não & 35,62 & 1,00 & $<0,001$ & 1,00 & 0,813 \\
\hline $\operatorname{Sim}$ & 50,0 & $1,40(1,20-1,72)$ & & $0.96(0,80-1,13)$ & \\
\hline \multicolumn{6}{|l|}{ Sesta } \\
\hline Nunca & 28,61 & 1,00 & $<0,001$ & 1,00 & 0,013 \\
\hline Às vezes & 43,05 & $1,50(1,26-1,85)$ & & $1,25(1,05-1,49)$ & \\
\hline Sempre & 66,67 & $2,33(1,82-3,05)$ & & $1,40(1,07-1,84)$ & \\
\hline
\end{tabular}

a Valor do $p$ não ajustado ( $p$ do Qui-quadrado)

${ }^{b}$ Valor do $p$ de tendência ajustado

\section{DISCUSSÃO}

As associações entre os padrões de sono, o nível socioeconômico e as condições de vida são relevantes, especialmente, para a formulação de políticas públicas e educacionais para saúde de crianças e adolescentes e carecem de melhores esclarecimentos. Apesar disso, a diminuição das horas de sono, independente da classe social, em crianças e adolescentes, é preocupante ${ }^{7,11,18}$. Javaheri et al. ${ }^{11}$, investigando jovens norteamericanos de 13 a 16 anos, verificaram que, mesmo após ajustes por sexo, estado nutricional, nível socioeconômico e comorbidades, a baixa duração do sono e a qualidade ruim do sono estiveram associadas, por exemplo, a maiores prevalências de hipertensão.

A duração média de sono observada neste estudo foi semelhante à da investigação com jovens de São Paulo realizada por Teixeira et al. ${ }^{23}$ que identificou uma duração média de sono de 8,8 horas para adolescentes não trabalhadores. Também foi semelhante à duração encontrada em estudantes do ensino médio da Nova Zelândia $\left(8,6\right.$ horas) ${ }^{24}$. No entanto, a prevalência de indivíduos que dormem oito ou menos horas encontrada nos jovens de São Paulo $(39,0 \%)$ foi menor que a observada em jovens argentinos $(49,0 \%)^{18}$. Embora não se saiba, com precisão, qual a necessidade média de sono na adolescência, muitos jovens necessitam mais de 9 horas de sono 25,26 .

A principal variável associada a menos de 8 horas de sono nos adolescentes investigados foi a idade, e os jovens apresentaram uma diminuição das horas de sono com o avanço da adolescência. Mudanças nos comportamentos de sono na adolescência devem-se tanto a um aumento das obrigações escolares, atividades sociais e inserção do mundo do trabalho como atividades como televisão e internet que podem colaborar para que os adolescentes deitem-se mais tarde e fiquem mais sonolentos ${ }^{27}$. Além disso, fatores maturacionais levam os adolescentes a apresentarem uma diminuição nas horas de sono, em razão de uma maior lentidão na inibição da secreção de melatonina no início da fase clara do dia, especialmente, nas fases tardias da puberdade, bem como uma acumulação mais lenta da propensão para o sono durante o dia ${ }^{27}$.

Os dados analisados mostraram uma tendência menos evidente de diminuição de horas de sono durante a adolescência nas classes socioeconômicas mais baixas. Embora uma descrição semelhante não tenha sido encontrada na literatura consultada, Moore e Meltzer ${ }^{4}$ apontam que existe uma importante interdependência entre nível educacional e socioeconômico, qualidade do sono e indicadores gerais de 
saúde. Na literatura consultada, os estudos com diferentes etnias são os que mais se aproximaram desse contexto.

A literatura ${ }^{5,6}$ aponta que, de forma geral, os jovens menos favorecidos apresentaram uma tendência de pior qualidade do sono e isso está associado, entre outros fatores, às condições ruins do ambiente de morar e dormir. Por outro lado, estudos também apontam para a associação entre baixa duração do sono com mais horas em frente à televisão, assistir à televisão à noite, uso de videogame e internet que poderiam estar associados, em certas realidades, a um maior poder aquisitivo ${ }^{28}$, o que parece estar mais coerente com os resultados encontrados em São Paulo.

Os resultados com os jovens de São Paulo apontaram para uma duração do sono maior nas classes mais baixas, motivada, especialmente, pelos horários de dormir mais tarde e acordar mais cedo da classe alta. Além disso, independente da classe socioeconômica, o turno escolar mostrou-se uma variável fortemente associada à duração do sono, confirmando dados da literatura que apontam que os adolescentes em dias com aula, especialmente, quando estudam ou trabalham pela manhã, apresentam maior privação de sono ${ }^{14,18}$.

Outra variável importante ao se analisar o sono dos adolescentes em diferentes níveis socioeconômicos é o envolvimento com o mundo do trabalho. Embora o trabalho não tenha sido uma variável significativa na análise ajustada e não terem sido analisados o turno e a duração deste, houve uma tendência de menor duração do sono nos adolescentes trabalhadores em todas as classes sociais, confirmando dados da literatura ${ }^{23,29}$. Além disso, o envolvimento com o trabalho pode ter levado os adolescentes da classe baixa, por exemplo, a manterem certa regularidade no horário de dormir no final da adolescência.

Em amostras brasileiras, não foram encontrados dados populacionais sobre a quantidade de sono em diferentes níveis socioeconômicos. $O$ estudo de Petry et al. ${ }^{30}$ se propôs a analisar questões relacionadas ao sono em jovens de classes baixas. Os autores discutiram, com base nos resultados de análises de jovens do município de Uruguaiana, RS, que baixos níveis socioeconômicos podem estar associados a maiores aglomerações familiares, tabagismo passivo e maiores incidências de infecções de vias aéreas, podendo aumentar a chance de surgimento de problemas respiratórios e do sono. Entretanto, o estudo não apresentou o controle da classe socioeconômica ou renda familiar e os autores partiram do pressuposto de que a população do município, no geral, apresentava baixo nível socioeconômico.

Os estudos com amostras estrangeiras apontaram, de forma geral, para o contrário do observado em São Paulo, um maior risco de diminuição da duração e da qualidade de sono nas classes menos favorecidas. Roberts et al., ${ }^{5,6}$, investigando adolescentes norte-americanos de diferentes origens étnicas, verificaram, por exemplo, que jovens com nível so- cioeconômico baixo apresentaram prevalências superiores de sonolência.

Segundo Buckhalt et al. ${ }^{3}$, crianças e adolescentes com menor nível socioeconômico apresentam maiores chances de dividir o quarto, maior número de pessoas morando na residência, dieta ruim e ambiente de dormir inadequado, o que pode levar a uma pior qualidade do sono. Neste mesmo contexto, DeSantis et al. ${ }^{10}$ sugerem que, além dos fatores genéticos, uma maior exposição ao estresse pré-natal, baixo peso ao nascer, experiências adversas na infância, experiências com racismo e discriminação podem ser fatores relevantes para o comportamento dos ritmos biológicos.

De forma geral, embora não se tenha encontrado uma descrição da duração de sono e do atraso de fase em diferentes níveis socioeconômicos, os resultados do presente trabalho apontam para possíveis particularidades dos jovens brasileiros quando comparados, especialmente, com populações norte-americanas em estudos que, normalmente, associam um nível socioeconômico mais baixo às etnias de origens africanas. Possíveis causas dessas diferenças podem estar relacionadas a uma menor segregação racial nas escolas públicas em que foram conduzidas as coletas e, também, ao menor percentual de jovens da classe alta nessas escolas.

Além da idade, do nível socioeconômico e do turno escolar, o hábito da sesta também se apresentou como um fator importante para a diminuição da quantidade de sono noturno. Uma diminuição das horas de sono, neste caso, pode ser explicada pelos mecanismos homeostáticos de regulação do sono ${ }^{12}$. A ocorrência da sesta atrasaria a propensão ao sono noturno, retardando seu início.

As principais limitações deste estudo referem-se ao desenho da investigação e à análise de algumas variáveis. O não acompanhamento longitudinal dos hábitos de sono impede possíveis análises de causa e efeito, permitindo apenas uma discussão de associações. Além disso, inúmeras variáveis, como problemas psiquiátricos e clínicos, utilização de medicamentos, ingestão de alguns alimentos, o turno e a duração do trabalho, entre outras, podem influenciar nos comportamentos de sono dos adolescentes e devem ser analisadas com maior profundidade em futuros estudos.

\section{CONCLUSÕES}

Das variáveis investigadas, os principais fatores associados a oito ou menos horas de sono na adolescência foram a idade, o nível socioeconômico, o turno escolar e o hábito da sesta. Os adolescentes com maior nível socioeconômico apresentaram uma tendência de menor duração do sono, dormindo mais tarde e acordando mais cedo que os demais grupos. Os jovens dos níveis socioeconômicos mais baixos apresentaram um atraso nos horários de dormir menos evidente com o avanço da adolescência. Recomendam-se análises mais detalhadas 
sobre os padrões de sono no decorrer da adolescência em jovens de diferentes níveis socioeconômicos, especialmente, com análises de fatores de confusão como raça, número de pessoas no quarto e na casa, hábitos alimentares, estado nutricional, problemas respiratórios e psiquiátricos, entre outros.

\section{REFERÊNCIAS}

1. IBGE. Instituto Brasileiro de Geografia e Estatística. Pesquisa de orçamentos familiares no Brasil, 2002/2003. Antropometria e análise do estado nutricional de crianças e adolescentes no Brasil. Rio de Janeiro; 2006.

2. Batista-Filho M, Miglioli TC, Santos MC. Normalidade antropométrica de adultos: o paradoxo geográfico e socioeconômico da transição nutricional no Brasil. Rev Bras Saúde Matern Infant. 2007;7(4):487-93.

3. Buckhalt JA, El-Sheikh M, Keller P. Children's sleep and cognitive functioning: race and socioeconomic status as moderators of effects. Child Dev. 2007;78(1):213-31.

4. Moore M, Meltzer LJ. The sleepy adolescent: causes and consequences of sleepiness in teens. Paediatr Respir Rev. 2008;9(2):114-20.

5. Roberts RE, Lee ES, Hemandez M, Solari AC. Symptoms of insomnia among adolescents in the lower Rio Grande Valley of Texas. Sleep. 2004;27(4):751-60

6. Roberts RE, Roberts CR, Chan W. Ethnic differences in symptoms of insomnia among adolescents. Sleep. 2006;29(3):359-65.

7. Roberts RE, Roberts CR, Duong HT. Sleepless in adolescence: prospective data on sleep deprivation, health and functioning. J Adolesc. 2009. [no prelo]

8. Paine SJ, Gander PH, Harris R, Reid P. Who reports insomnia? Relationships with age, sex, ethnicity, and socioeconomic deprivation. Sleep. 2004;27(6):1163-9.

9. McLaughlin Crabtree V, Beal Korhonen J, Montgomery-Downs HE, Faye Jones V, O'Brien LM, Gozal D. Cultural influences on the bedtime behaviors of young children. Sleep Med. 2005;6(4):319-24.

10. DeSantis AS, Adam EK, Doane LD, Mineka S, Zinbarg RE, Craske MG. Racial/ethnic differences in cortisol diurnal rhythms in a community sample of adolescents. J Adolesc Health. 2007:41(1):3-13.

11. Javaheri S, Storfer-Isser A, Rosen CL, Redline S. Sleep quality and elevated blood pressure in adolescents. Circulation. 2008:118(10):1034-40.

12. Carskadon MA, Viera C, Acebo C. Association between puberty and delayed phase preference. Sleep. 1993;16(3):258-62.

13. Wolfson AR, Carskadon MA. Sleep schedules and daytime functioning in adolescents. Child Dev. 1998;69(4):875-87.
14. Thorleifsdottir B, Björnsson JK, Benediktsdottir B, Gislason T, Kristbjarnarson H. Sleep and sleep habits from childhood to young adulthood over a 10-year period. J Psychosom Res. 2002:53(1):529-37.

15. Hitze B, Bosy-Westphal A, Bielfeldt F, Settler U, Plachta-Danielzik S, Pfeuffer M, et al. Determinants and impact of sleep duration in children and adolescents: data of the Kiel Obesity Prevention Study. Eur J Clin Nutr. 2009;63(6):739-46.

16. Pereira EF, Teixeira CS, Louzada FM. Daytime sleepiness in adolescents: prevalences and associated factors. Rev Paul Pediatr. 2010. [no prelo]

17. Gaina A, Sekine M, Hamanishi S, Chen X, Wang H, Yamagami T, et al. Daytime sleepiness and associated factors in Japanese school children. J Pediatr. 2007;151(5):518-22.

18. Perez-Chada D, Perez-LloretS, Videla AJ, Cardinali D, Bergna MA, Fernández-Acquier M, et al. Sleep disordered breathing and daytime sleepiness are associated with poor academic performance in teenagers. A study using the Pediatric Daytime Sleepiness Scale (PDSS). Sleep. 2007;30(12):1698-703.

19. Louzada FM, Menna-Barreto L. Sleep-wake cycle in rural populations. Biol Rhythm Res. 2004;35(1/2):153-7.

20. ABEP. Associação Brasileira de Empresas de Pesquisa. Critério de Classificação Econômica Brasil; 2003. Disponível em: http://www.abep.org. Acesso em: 20/6/2005.

21. Spiegelman D, Hertzmark E. Easy SAS calculations for risk or prevalence ratios and differences. Am J Epidemiol. 2005;162:199-200.

22. Hosmer DW, Leneshow S. Applied logistic regression. New York: Wiley; 1989

23. Teixeira LR, Lowden A, Turte SL, Nagai R, Moreno CRC, Latorre MRDO, et al. Sleep and sleepiness among working and non-working high school evening students. Chronobiol Int. 2007;24(1):99-113.

24. Dorofaeff TF, Denny S. Sleep and adolescence. Do New Zealand teenagers get enough? J Paediatr Health. 2006;42(9):515-20.

25. Loessl B, Valerius G, Kopasz M, Hornyak M, Riemann D, Voderholzer U. Are adolescents chronically sleep-deprived? An investigation of sleep habits of adolescents in the Southwest of Germany. Child Care Health Dev. 2008;34(5):549-56.

26. Wolfson AR, Spaulding NL, Dandrow C, Baroni EM. Middle school start times: the importance of a good night's sleep for young adolescents. Behav Sleep Med. 2007;5(3):194-209.

27. Carskadon MA, Acebo C, Jenni CC. Regulation of adolescent sleep: implications for behavior. Ann N Y Acad Sci. 2004;1021:276-91.

28. BaHammam A, Bin Saeed A, Al-Faris E, Shaikh S. Sleep duration and its correlates in a sample of Saudi elementary school children. Singapore Med J. 2006;47(10):875-81.

29. Fischer FM, Nagai R, Teixeira LR. Explaining sleep duration in adolescents: the impact of socio-demographic and lifestyle factors and working status. Chronobiol Int. 2008;25:359-72

30. Petry C, Pereira UM, Pitrez PM, Jones MH, Stein RT. The prevalence of symptoms of sleepdisordered breathing in Brazilian schoolchildren. J Pediatr. 2008;84(2):123-9. 